\title{
Instability of hyper-compact Kerr-like objects
}

\author{
Vitor Cardosd* \\ Centro Multidisciplinar de Astrofísica - CENTRA, Dept. de Física, \\ Instituto Superior Técnico, Av. Rovisco Pais 1, 1049-001 Lisboa, Portugal \\ and Department of Physics and Astronomy, The University of Mississippi, University, MS 38677-1848, USA \\ Paolo Pan用 and Mariano Cadon \\ Dipartimento di Fisica, Università di Cagliari, and INFN sezione di Cagliari, Cittadella Universitaria 09042 Monserrato, Italy \\ Marco Cavagliàs \\ Department of Physics and Astronomy, The University of Mississippi, University, MS 3867r-1848, USA
}

(Dated: October 23, 2018)

\begin{abstract}
Viable alternatives to astrophysical black holes include hyper-compact objects without horizon, such as gravastars, boson stars, wormholes and superspinars. The authors have recently shown that typical rapidly-spinning gravastars and boson stars develop a strong instability. That analysis is extended in this paper to a wide class of horizonless objects with approximate Kerr-like geometry. A detailed investigation of wormholes and superspinars is presented, using plausible models and mirror boundary conditions at the surface. Like gravastars and boson stars, these objects are unstable with very short instability timescales. This result strengthens previous conclusions that observed hyper-compact astrophysical objects with large rotation are likely to be black holes.
\end{abstract}

PACS numbers: 04.40.Dg,04.30.Nk,04.25.Nx,95.85.Sz,04.70.-s

\section{INTRODUCTION}

Astrophysical Black Holes (BHs) are believed to be common objects in galaxies. Their mass is expected to span many orders of magnitude, from a fraction of the solar mass (primordial BHs in the galactic halo) to few solar masses (stellar BHs in the galactic plane) up to several billions of solar masses (supermassive BHs in galactic centers). Their angular momentum should be close to the extremal limit due to accretion and mergers 1, 2]. For example, if quasars are powered by supermassive BHs, astrophysical observations suggest that they should be rotating near the Kerr bound [3].

Unquestionable observational evidence of the existence of BHs is still lacking [4, [5, [6]. Current astrophysical data cannot rule out "BH forgeries", i.e. hyper-compact objects with redshift and geodesics similar to those of BHs, but lacking an event horizon. Several models of hyper-compact objects with these characteristics have been known in the literature for some time. Among these models, gravastars [7, 8] and boson stars [9, 10] have been proposed as the most viable alternatives to astrophysical BHs. The authors recently showed that rapidly spinning gravastars and boson stars may develop a strong ergoregion instability [11]. Their typical instability timescales are of order of 0.1 seconds to 1 week for objects with mass $M=1-10^{6} M_{\odot}$. Therefore, observed astrophysical hyper-compact objects are likely not to be gravastars nor boson stars.

The purpose of this paper is to compute the ergoregion instability for other horizonless, Kerr-like hyper-compact objects: wormholes and superspinars. Wormholes can be objects even simpler than BHs [12, 13, 14]. They are infinitesimal variations of the Schwarzschild space-time which may be indistinguishable from BHs [15]. In a string theory context, the fuzzball model replaces BHs by horizonless structures [16]. The BH-like geometry emerges in a coarse-grained description which "averages" over horizonless geometries and produces an effective horizon at a radius where the individual microstate geometries start to differ. Superspinars are solutions of the gravitational field equations that violate the Kerr bound. These geometries could be created by high energy corrections to Einstein gravity such as those present in string-inspired models [17, 18]. Superspinars are expected to have compactness of the order of extremal rotating Kerr BHs and to exist in any mass range.

\footnotetext{
${ }^{*}$ Electronic address: vcardoso@fisica.ist.utl.pt

${ }^{\dagger}$ Electronic address: paolo.pani@ca.infn.it

‡Electronic address: mariano.cadoni@ca.infn.it

$\S$ Electronic address: cavaglia@phy.olemiss.edu
} 
A rigorous analysis of the ergoregion instability for these models is a non-trivial task; known wormhole solutions are special non-vacuum solutions of the gravitational field equations. Thus their investigation requires a case-by-case analysis of the stress-energy tensor. Exact solutions of four-dimensional superspinars are not known. To overcome these difficulties, the following analysis will focus on a simple model which captures the essential features of most Kerr-like horizonless hyper-compact objects. Superspinars and rotating wormholes will be modeled by the exterior Kerr metric down to their surface, where Dirichlet boundary conditions are imposed. This problem is very similar to Press and Teukolsky's "BH bomb" [19, 20], i.e. a rotating BH surrounded by a perfectly reflecting mirror with its horizon replaced by a reflecting surface. These boundary conditions are perfect mirror conditions and require a reflection coefficient $R=1$. In a more realistic model $R<1$ and a certain transmittance $T=1-R$ should be taken into account, which will in principle decrease the strength of the ergoregion instability. We argue that the qualitative behavior of the instability is the same as long as the reflection or the superradiant amplification are large enough. Letting $\rho$ be a superradiant factor, one expects an ergoregion instability to develop whenever $\rho(1-T)>1$. In the perfect mirror limit $T=0$ and the superradiant condition is simply $\rho>1$. The general case can be handled using both the analytical and numerical techniques presented here.

In Section 【 we introduce the class of objects we will deal with in this work. They are general approximations to superspinars and wormholes with the basic key features retained. In Section III we show how to solve for the instability analytically in two different regimes. The details of these computations are left for Appendices $\mathrm{A}$ and $\mathrm{B}$, These approximations are compared with numerical results in Section IV] where we also show that another kind of instability sets in for general naked singularities. This "algebraic" instability can be computed algebraically in the Kerr geometry. We close with a brief discussion of our results.

\section{SUPERSPINARS AND KERR-LIKE WORMHOLES}

A superspinar of mass $M$ and angular momentum $J=a M$ can be modeled by the Kerr geometry [17]

$$
d s_{\text {Kerr }}^{2}=-\left(1-\frac{2 M r}{\Sigma}\right) d t^{2}+\frac{\Sigma}{\Delta} d r^{2}+\left[\left(r^{2}+a^{2}\right) \sin ^{2} \theta+\frac{2 M r}{\Sigma} a^{2} \sin ^{4} \theta\right] d \phi^{2}-\frac{4 M r}{\Sigma} a \sin ^{2} \theta d \phi d t+\Sigma d \theta^{2},
$$

where

$$
\Sigma=r^{2}+a^{2} \cos ^{2} \theta, \Delta=r^{2}+a^{2}-2 M r .
$$

Unlike Kerr BHs, superspinars have $a>M$ and no horizon. Since the domain of interest is $-\infty<r<+\infty$, the space-time posesses naked singularities and closed timelike curves in regions where $g_{\phi \phi}<0$ (see the monograph by Chandrasekhar [34]). High energy modifications in the vicinity of the singularity are also expected. Following Ref. [17], a small region around the origin is excised or assumed to be modified by, say, stringy corrections. The most popular excision method uses domain walls formed by supertubes [17, 21]. Kerr-like wormholes are described by metrics of the form

$$
d s_{\text {wormhole }}^{2}=d s_{\text {Kerr }}^{2}+\delta g_{a b} d x^{a} d x^{b},
$$

where $\delta g_{a b}$ is infinitesimal. In general, Eq. (2.3) describes an horizonless object with excision at some small distance of order $\epsilon$ from the would-be horizon. (See Ref. [15] for details on nonrotating wormholes). A detailed consideration of rotating stationary wormholes, throat location and conditions on the metric is given by Teo [22] to which we refer for further details. Here, we simply assume these conditions are satisfied. Wormholes require exotic matter and/or divergent stress tensors, thus some ultra-stiff matter is assumed close to the would-be horizon. In the following, both superspinars and wormholes will be modeled by the Kerr metric with a rigid "wall" at finite Boyer-Lindquist radius $r_{0}$, which excludes the pathological region. We will consider both $a / M<1$ and $a / M>1$.

If the background geometries of superspinars and wormholes are sufficiently close to the Kerr geometry, their dynamical perturbations are determined by the equations of perturbed Kerr BHs. The proof is straightforward. Consider a minimally-coupled scalar field $\Phi$ propagating on a space-time with metric

$$
g_{a b}=g_{a b}^{0}+\delta g_{a b},
$$

where $\delta g_{a b} \ll g_{a b}$. At first order in $\delta g_{a b}$, the Klein-Gordon equation reads

$$
\partial_{a}\left[\sqrt{-g} g^{a b} \partial_{b} \Phi\left(1+\frac{1}{2} g^{c d} \delta g_{c d}\right)\right]+\partial_{a}\left[\sqrt{-g} \delta g^{a b} \partial_{b} \Phi\left(1+\frac{1}{2} g^{c d} \delta g_{c d}\right)\right]=0 .
$$

If $g^{c d} \delta g_{c d} \ll 1$, Eq. (2.5) is identical to the equation of a scalar field in the Kerr geometry. This result also generalizes to the Maxwell field. Gravitational perturbations can be handled as in the Kerr geometry only if they are larger than $\delta g_{a b}$ at any time. 


\section{INSTABILITY ANALYSIS: ANALYTIC RESULTS}

The instability of superspinars and wormholes is studied by considering Kerr geometries with arbitrary rotation parameter $a$ and a "mirror" at some Boyer-Lindquist radius $r_{0}$. Using the Kinnersley tetrad and Boyer-Lindquist coordinates, it is possible to separate the angular variables from the radial ones, decoupling all quantities. Small perturbations of a spin- $s$ field are reduced to the radial and angular master equations [23]

$$
\begin{gathered}
\Delta^{-s} \frac{d}{d r}\left(\Delta^{s+1} \frac{d R_{l m}}{d r}\right)+\left[\frac{K^{2}-2 i s(r-M) K}{\Delta}+4 i s \omega r-\lambda\right] R_{l m}=0, \\
{\left[\left(1-x^{2}\right)_{s} S_{l m, x}\right]_{, x}+\left[(a \omega x)^{2}-2 a \omega s x+s+{ }_{s} A_{l m}-\frac{(m+s x)^{2}}{1-x^{2}}\right]{ }_{s} S_{l m}=0,}
\end{gathered}
$$

where $x \equiv \cos \theta, \Delta=r^{2}-2 M r+a^{2}, K=\left(r^{2}+a^{2}\right) \omega-a m$ and the separation constants $\lambda$ and ${ }_{s} A_{l m}$ are related by

$$
\lambda \equiv{ }_{s} A_{l m}+a^{2} \omega^{2}-2 a m \omega .
$$

If $a \leqslant M$, the space-time possesses one or two horizons located at $r_{ \pm}=M \pm \sqrt{M^{2}-a^{2}}$. Equations (3.1)-(3.2) can be analytically solved in the slowly-rotating and low-frequency regime, $\omega M \ll 1$ [20, 24, 25], and in the rapidly-spinning regime, where $r_{+} \sim r_{-}$and $\omega \sim m \Omega_{h}, \Omega_{h} \equiv a /\left(2 M r_{+}\right)$being the angular velocity at the horizon [25].

\section{A. Slowly rotating objects and low frequencies}
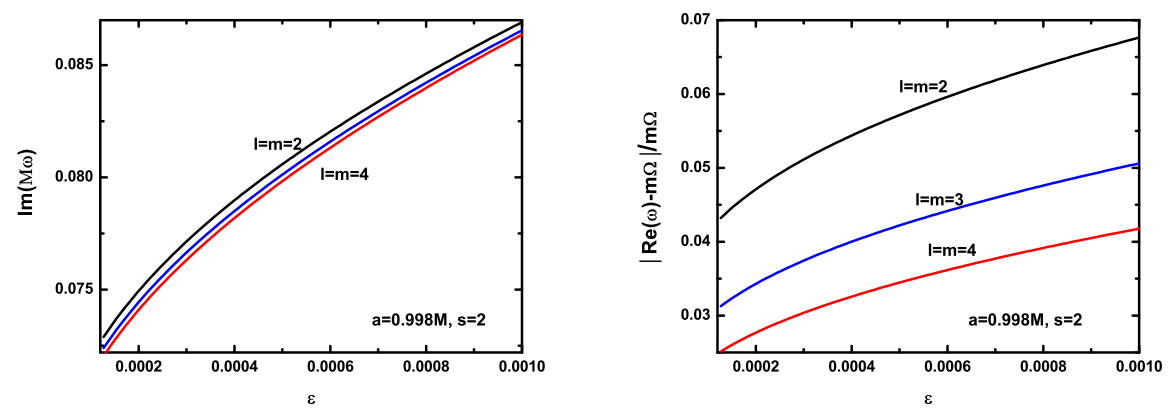

FIG. 1: Imaginary and real parts of the characteristic gravitational frequencies for an object with $a=0.998 M$, according to the analytic calculation for rapidly-spinning objects. The mirror location is at $r_{0}=(1+\epsilon) r_{+}$. The real part is approximately constant and close to $m \Omega$, in agreement with the assumptions used in the analytic approach.

The instability timescales for small rotation and low frequencies can be computed by approximating the Teukolsky equation near the horizon and then matching its solution to the solution near infinity. The spheroidal wavefunctions (3.2) reduce to the spin-weighted spherical harmonics with eigenvalues ${ }_{s} A_{l m}=l(l+1)-s(s+1)$. Matching the inner solution to the outer solution, the frequency $\omega$ is (see Appendix $\mathrm{A}$ for details)

$$
z_{0}^{-s-2 i \varpi}=\prod_{k=1}^{l}\left(\frac{k+s+2 i \varpi}{k-s-2 i \varpi}\right)\left[\frac{1-i\left(\omega\left(r_{+}-r_{-}\right)\right)^{2 l+1} L_{s}(s+2 i \varpi) \prod_{k=1}^{l}\left(k^{2}+4 \varpi^{2}-s^{2}-4 i s \varpi\right)}{1+i\left(\omega\left(r_{+}-r_{-}\right)\right)^{2 l+1} L_{s}(s+2 i \varpi) \prod_{k=1}^{l}\left(k^{2}+4 \varpi^{2}-s^{2}-4 i s \varpi\right)}\right]
$$

where

$$
L_{s}=2^{2 l-1}(-1)^{s} \Gamma(1+l-s) \Gamma(1+l+s)\left[\frac{\Gamma(l+1)}{\Gamma(2 l+2) \Gamma(2 l+1)}\right]^{2} .
$$

Equation (3.4) can be solved numerically for the characteristic values of $\omega$. Care must be exercised to ensure that the solutions are consistent with the approximation scheme, i.e., $M \omega \ll 1, a \ll M$ and $z_{0} \sim 0$. The instability timescale 
for scalar perturbations is

$$
\frac{\tau}{M}=2\left[\frac{1-(a / \sqrt{2} M)^{2}+\sqrt{1-(a / M)^{2}}}{\sqrt{1-(a / M)^{2}}}\right] \cdot \log ^{-1}\left[\frac{1+\gamma\left(\omega_{n, m}-m \Omega\right)}{1-\gamma\left(\omega_{n, m}-m \Omega\right)}\right] .
$$

Table \shows the results for $s=0$ and different values of $l=m$. The scalar instability timescale is much smaller

TABLE I: Approximate solution for a slowly rotating object with $a=0.3 M$ and mirror position at $\epsilon=10^{-5}$. The two columns give the real part of the frequency $\operatorname{Re}(\omega)$ and the growth time $\tau$ for different $l=m$ values of the scalar field, respectively. The mode $l=m=1$ is absent.

\begin{tabular}{|c||cc|}
\hline \multicolumn{3}{|c|}{$a / M=0.3, \epsilon=10^{-5}$} \\
\hline$l=m$ & $\operatorname{Re}(\omega) M$ & $\tau / M$ \\
2 & 0.02 & $1.8 \times 10^{13}$ \\
3 & 0.09 & $5.8 \times 10^{13}$ \\
4 & 0.17 & $8.1 \times 10^{15}$ \\
\hline \hline
\end{tabular}

than the Hawking evaporation timescale [26], $\tau_{\text {evap }} \approx 10^{71}\left(M / M_{\odot}\right)^{3}$ sec, and increases with $l=m$. Gravitational instability timescales of fast rotating objects are expected to be larger by several orders of magnitude.

\section{B. Fast-rotating objects}

Defining $x=\left(r-r_{+}\right) / r_{+}, \sigma=\left(r_{+}-r_{-}\right) / r_{+}, \tau=M(\omega-m \Omega)$ and $\omega^{\prime} \rightarrow r_{+} \omega$, the Teukolsky equation can be written as

$$
\left[2 i \omega^{\prime} x^{2}+x\left(4 i \omega^{\prime}-2(s+1)\right)-(s+1) \sigma+4 i \tau\right] R^{\prime}(x)+\left[2(2 s+1) i \omega^{\prime}(x+1)+\lambda\right] R(x)-x(x+\sigma) R^{\prime \prime}(x)=0 .
$$

Equation (3.7) can be solved in the limit $a \rightarrow M$ and $\omega \rightarrow m \Omega$ by following the procedure of the previous section. The relation between the position of the mirror and the frequency of the wave is (see Appendix B for details)

$$
\left(-z_{0}\right)^{-s-i \kappa}=\frac{R_{1}+\rho R_{3}(-2 i \omega \sigma)^{2 i \delta}}{R_{2}+\rho R_{4}(-2 i \omega \sigma)^{2 i \delta}} .
$$

Numerical solutions of the above equation for a star with $a=0.998 M$ are shown in Fig. 1. The real part of the characteristic frequency is always close to $m \Omega$ and $\operatorname{Im}(\omega) \ll \operatorname{Re}(\omega)$. Thus the results are consistent with the initial assumptions. The instability timescale for gravitational perturbations is about five orders of magnitude smaller than the instability timescale for scalar perturbations.

\section{INSTABILITY ANALYSIS: NUMERICAL RESULTS}

The oscillation frequencies of the modes can be found from the canonical form of Eq. (3.1)

$$
\frac{d^{2} Y}{d r_{*}^{2}}+V Y=0
$$

where

$$
Y=\Delta^{s / 2}\left(r^{2}+a^{2}\right)^{1 / 2} R, \quad V=\frac{K^{2}-2 i s(r-M) K+\Delta(4 i r \omega s-\lambda)}{\left(r^{2}+a^{2}\right)^{2}}-G^{2}-\frac{d G}{d r_{*}}
$$

and $K=\left(r^{2}+a^{2}\right) \omega-a m, G=s(r-M) /\left(r^{2}+a^{2}\right)+r \Delta\left(r^{2}+a^{2}\right)^{-2}$. The separation constant $\lambda$ is related to the eigenvalues of the angular equation by Eq. (3.3). The eigenvalues ${ }_{s} A_{l m}$ are expanded in power series of $a \omega$ as [27]

$$
{ }_{s} A_{l m}=\sum_{k=0} f_{s l m}^{(k)}(a \omega)^{k} .
$$

Terms up to order $(a \omega)^{2}$ are included in the calculation. Absence of ingoing waves at infinity implies

$$
Y \sim r^{-s} e^{i \omega r_{*}} .
$$


Numerical results are obtained by integrating Eq. (4.1) inward from a large distance $r_{\infty}$. The integration is performed with the Runge-Kutta method with fixed $\omega$ starting at $M r_{\infty}=400$, where the asymptotic behavior (4.4) is imposed. (Choosing a different initial point does not affect the final results.) The numerical integration is stopped at the radius of the mirror $r_{0}$, where the value of the field $Y\left(\omega, r_{0}\right)$ is extracted. The integration is repeated for different values of $\omega$ until $Y\left(\omega, r_{0}\right)=0$ is obtained with the desired precision. If $Y\left(\omega, r_{0}\right)$ vanishes, the field satisfies the boundary condition for perfect reflection and $\omega=\omega_{0}$ is the oscillation frequency of the mode.

\section{A. Objects with $a<M$}

The regime $a<M$ requires a surface or mirror at $r_{0}=r_{+}(1+\epsilon)>r_{+}$. Typical results for scalar perturbations of objects with $a<M$ are summarized in Table \ and Fig. 2. The top panels of Fig. 2 show the imaginary and real parts of the fundamental mode frequency for $a=0.998 M$ vs. the mirror position $r_{0}=r_{+}(1+\epsilon)$ for different $l=m$ values, respectively. The instability is weaker for larger $m$. This result holds also for $l \neq m$, although it is not shown in the plots. Comparisons with the analytic results in the near-extremal regime are shown in the bottom panels. The numerical integrations indicate that $\operatorname{Re}(\omega) \sim m \Omega$, in agreement with the analytic results of Sect. IIIB. The instability timescales are consistent with the analytic results within a factor $\sim 3$. The minimum instability timescale is of order $\tau \sim 10^{5} M$ for a wide range of mirror locations.

TABLE II: Characteristic frequencies and instability timescales for a Kerr-like object with $a=0.998 M$. The mirror is located at $\epsilon=0.1$. Results for scalar and gravitational perturbations are shown for different $l=m$ values.

\begin{tabular}{|c||cc|}
\hline \multicolumn{3}{|c|}{$(\operatorname{Re}(\omega) M, \operatorname{Im}(\omega) M)$} \\
\hline$l=m$ & $s=0$ & $s=2$ \\
1 & $\left(0.1120,0.6244 \times 10^{-5}\right)$ & - \\
2 & $\left(0.4440,0.5373 \times 10^{-5}\right)$ & $(0.4342,0.2900)$ \\
3 & $\left(0.7902,0.1928 \times 10^{-5}\right)$ & $(0.7803,0.2977)$ \\
4 & $\left(1.1436,0.5927 \times 10^{-6}\right)$ & $(1.1336,0.3035)$ \\
\hline \hline
\end{tabular}

Figure 3 shows the results for gravitational perturbations. Instability timescales are of the order of $\tau \sim 2-6 M$. Thus gravitational perturbations lead to an instability about five orders of magnitude stronger than the instability due to scalar perturbations (see Table 【I). Figure 3 shows that the ergoregion instability remains relevant even for values of the angular momentum as low as $a=0.6 \mathrm{M}$.

Some features of these results are intriguing and deserve further study. For instance, the regime $\operatorname{Re}(\omega)>m \Omega$ may also be unstable. Since the superradiant instability is confined to the superradiant regime, this mode should describe a different kind of instability. Numerical results also show that there is a mirror location which maximizes the instability at fixed $a$. It would be interesting to explain the physical meaning of this location.

\section{B. Objects with $a>M$}

Objects with $a>M$ could potentially describe superspinars. Several arguments suggest that objects rotating above the Kerr bound are unstable. Firstly, extremal Kerr BHs are marginally stable. Thus the addition of extra rotation should lead to instability. Secondly, fast-spinning objects usually take a pancake-like form [28] and are subject to the Gregory-Laflamme instability [29, 30]. Finally, Kerr-like geometries, like naked singularities, seem to be unstable against a certain class of gravitational perturbations [31, 32, 33] called algebraically special perturbations [34]. These perturbations are described by modes with zero Teukolsky-Starobinsky constant [23], and will be discussed in more detail below. For objects with $a>M$ the surface or mirror can be placed anywhere outside $r=0$. In general the instability is as strong as in the $a<M$ regime. An example in shown in Fig. [4, which displays the resonant frequencies for the instability of a surface at $r_{0} / M=0.001$. This result seems to confirm other investigations suggesting that ultra-compact objects rotating above the Kerr bound are unstable [35].

\section{Algebraically special modes}

Naked singularities are also characterized by a further kind of instability which is not related to the presence of an ergoregion [31, 32, 33]. This instability is generated by modes with vanishing Teukolsky-Starobinsky constant [23]. 

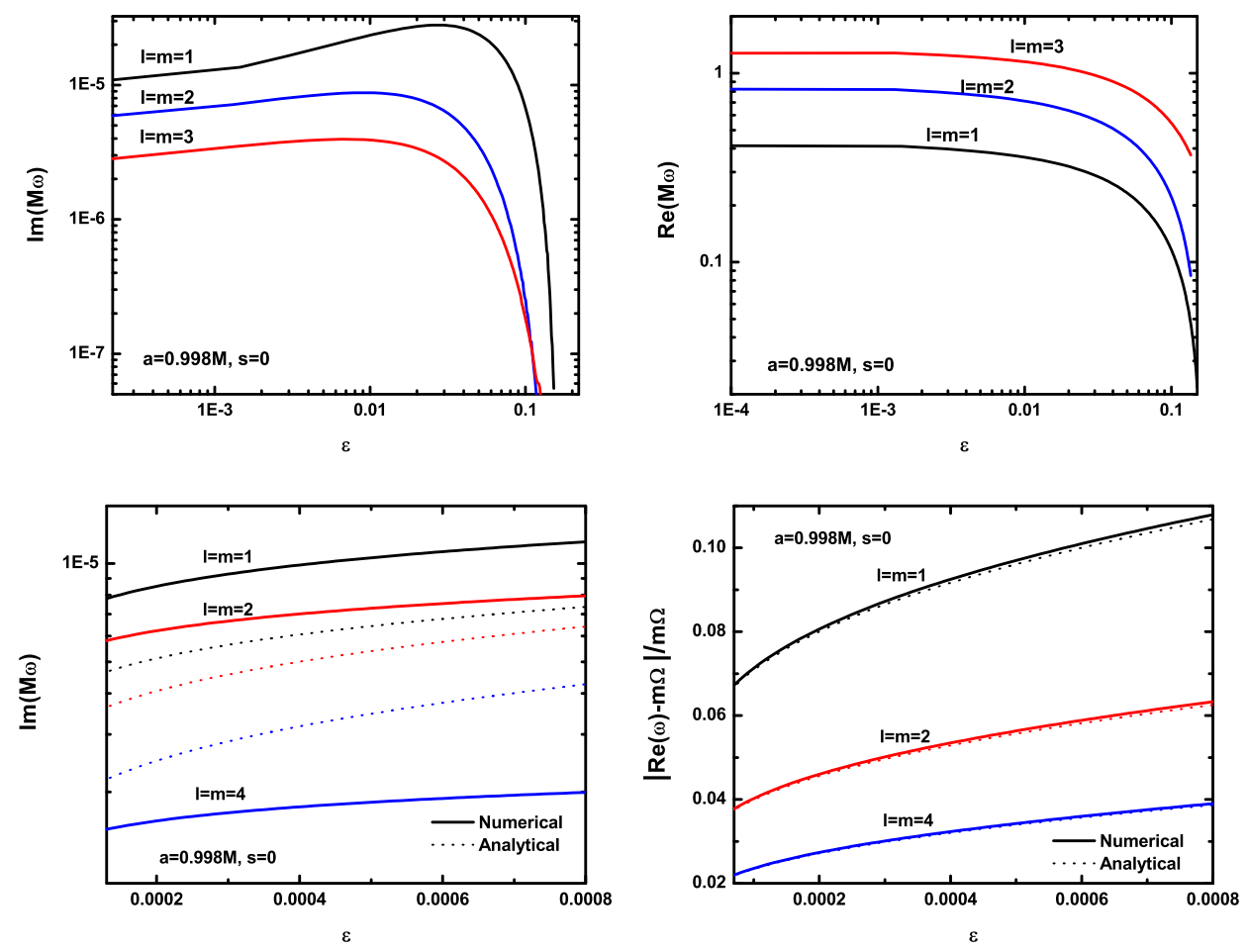

FIG. 2: Details of the instability for scalar perturbations, from a numerical solution of Teukolsky equation. Top panels: Numerical results for the imaginary part (left panel) and real part (right panel) of the fundamental mode frequency vs. the mirror position $r_{0}=r_{+}(1+\epsilon)$ for different $l=m$ values. The angular momentum is $a=0.998 M$. The instability grows monotonically with $l=m$. Bottom panels: Zoomed-in version of the upper panels, where numerical results (solid lines) are compared to the analytic solutions in the near-extremal regime (3.8) (dotted lines). The agreement between numerical and analytic values of the resonant frequency is remarkable. The analytic results for the imaginary part (bottom left panel) agree with the numerical results within a factor $\sim 3$.

These "algebraically special modes" are regular for $r>0$ and may be relevant to superspinar geometries without pathological regions. They can be expressed in analytic form. The solution of the Teukolsky equation is

$$
R_{l m}(r)=\left(A+B r+C r^{2}+D r^{3}\right) e^{i \omega^{\mathrm{AS}}\left(r_{*}-t\right)+i m \phi} S_{l m}(\theta),
$$

where $A, B, C, D$ are constants and $S_{l m}$ are spin weight-2 spheroidal harmonics [27]. These modes satisfy proper boundary conditions at infinity and are well behaved for any $r>-\infty$. Although superspinars require particular boundary conditions at the excised region, unstable algebraically special modes can be present. They can be computed with the continued fraction method [36, 37] and correspond to a zero of the Teukolsky-Starobinsky constant squared:

$$
|C|^{2}=\lambda^{2}(\lambda+2)^{2}-8 \omega^{2} \lambda\left[\alpha^{2}(6+5 \lambda)-12 a^{2}\right]+144 \omega^{2}\left(\alpha^{4} \omega^{2}+M^{2}\right),
$$

where $\lambda$ is defined in Eq. (3.3) for $s=-2$ and $\alpha^{2}=a^{2}-m a / \omega$. (Note a typographical error in Ref. 37.) The technique of Ref. [36] can be used to evaluate the algebraically special modes at fixed $a$. In the range $0<a<M$, the modes coincide with those of Ref. [37]. Some results for $a>M$ are listed in Table [II] The typical timescales are of the order of $10^{-6}$ seconds for a $1 M_{\odot}$ star and 1 second for $M=10^{6} M_{\odot}$.

\section{DISCUSSION}

This paper presented a general method for investigating the ergoregion instability of ultra-compact, horizonless Kerr-like objects. The essential features of these objects have been captured by a simple model whose physical properties are largely independent from the dynamical details of the gravitational system. The method has been applied to superspinars and rotating wormholes. Numerical and analytic results show that the ergoregion instability of these objects is extremely strong for any value of their angular momentum, with timescales of order $10^{-5}$ seconds 

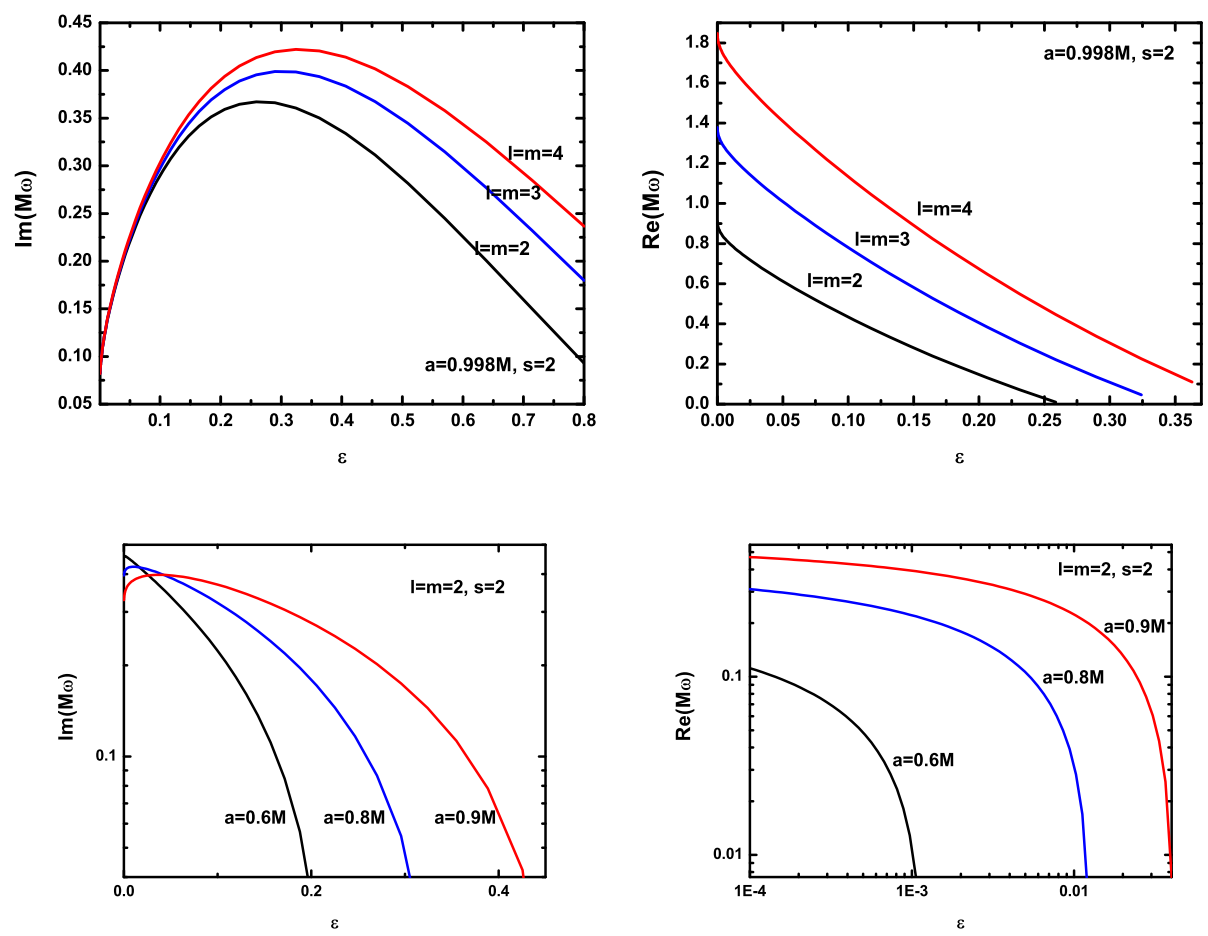

FIG. 3: Details of the instability for gravitational perturbations, for different $l=m$ modes and $a / M=0.998$ (top panels) and for $l=m=2$ and different $a / M<1$.
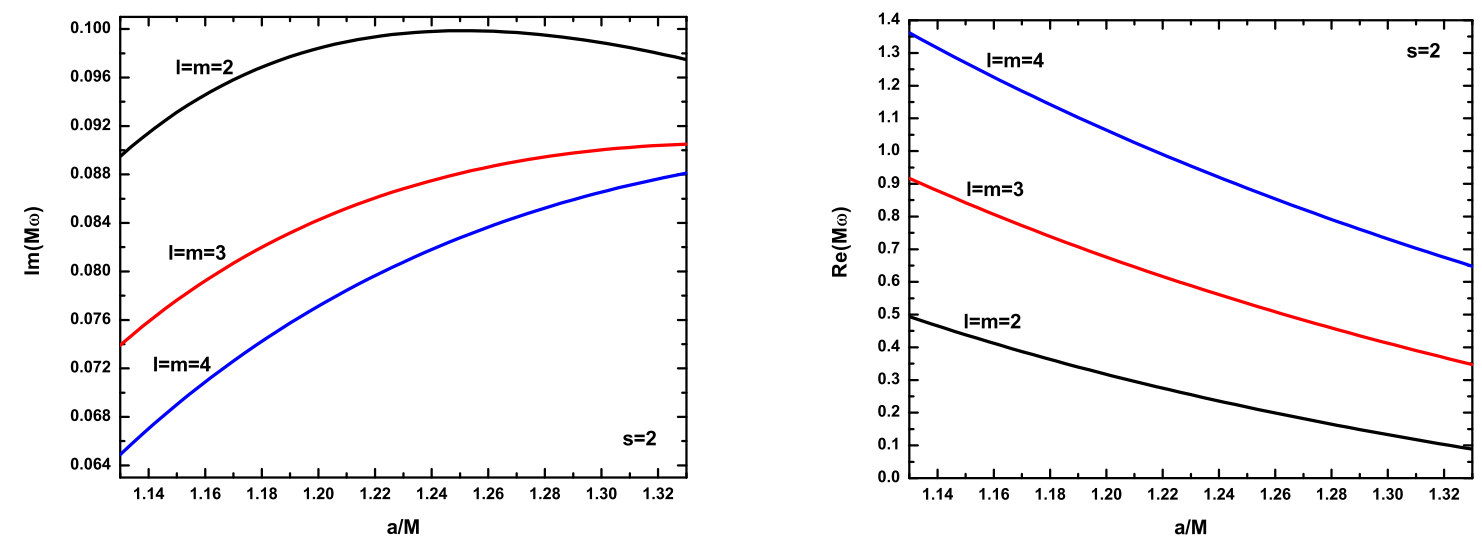

FIG. 4: The fundamental $l=m=2,3,4$ modes of an object spinning above the Kerr bound as function of rotation. The surface is located at $r_{0} / M=0.001$.

for a $1 M_{\odot}$ star and 10 seconds for a $M=10^{6} M_{\odot}$ star. The above investigation confirms previous results for gravastars and boson stars [11], namely that exotic objects without event horizon are likely to be ruled out as viable candidates for astrophysical hyper-compact objects. 


\begin{tabular}{|c|c|c|}
\hline \multicolumn{3}{|c|}{$2 M \omega$} \\
\hline$\frac{a}{M}$ & $\mathrm{~m}=0$ & $\mathrm{~m}=2$ \\
\hline 0.00 & $(0,4.0000)$ & $(0.0000,4.0000)$ \\
\hline 0.10 & $(0,4.03107)$ & $(0.918857,3.66121)$ \\
\hline 0.20 & $(0,4.13218)$ & $(1.36523,3.07054)$ \\
\hline 0.30 & $(0,4.33511)$ & $(1.51483,2.57005)$ \\
\hline 0.40 & $(0,4.74699)$ & $(1.53973,2.19077)$ \\
\hline 0.50 & $(0.715878,6.57057)$ & $(1.51441,1.90331)$ \\
\hline 0.60 & $(2.47766,5.25615)$ & $(1.46928,1.68066)$ \\
\hline 0.70 & $(2.84578,4.36141)$ & $(1.41732,1.50403)$ \\
\hline 0.80 & $(2.93170,3.71616)$ & $(1.36428,1.36084)$ \\
\hline 0.90 & $(2.91142,3.23046)$ & $(1.31270,1.24256)$ \\
\hline 1.00 & $(2.84632,2.85259)$ & $(1.26369,1.14328)$ \\
\hline 1.02 & $(2.83051,2.78693)$ & $(1.25424,1.12531)$ \\
\hline 1.04 & $(2.81414,2.72409)$ & $(1.24490,1.10790)$ \\
\hline 1.10 & $(2.76243,2.55079)$ & $(1.21763,1.05878)$ \\
\hline 1.20 & $(2.67192,2.30455)$ & $(1.17458,0.98601)$ \\
\hline 1.30 & $(2.58073,2.10007)$ & $(1.13444,0.92268)$ \\
\hline 1.40 & $(2.49185,1.92767)$ & $(1.09701,0.86707)$ \\
\hline
\end{tabular}

TABLE III: Algebraically special modes for various values of the angular momentum.

\section{Acknowledgements}

The authors are grateful to Matteo Losito for a careful reading of the manuscript. This work was partially funded by Fundação para a Ciência e Tecnologia (FCT) - Portugal through projects PTDC/FIS/64175/2006 and POCI/FP/81915/2007. One of the authors (MC) gratefully acknowledges the support of the National Science Foundation through LIGO Research Support grant NSF PHY-0757937.

\section{APPENDIX A: ANALYTIC SOLUTION IN THE LOW-FREQUENCY REGIME}

This appendix contains the analytic computation of the instability of rotating objects, bounded by a hard wall. Massless and massive scalar perturbations and general spin- $s$ perturbations are considered.

\section{Massless scalar fields}

Following Ref. [20, 24, 25], the space-time outside the star is divided in a near region, $r-r_{+} \ll 1 / \omega$, and a far region, $r-r_{+} \gg M$. The radial equation (3.1) is solved separately in each of these two regions with the assumptions that the Compton wavelength of the scalar particle is much larger than the typical size of the object, $1 / \omega \gg M$, and $a \ll M$. These solutions are then matched in the overlapping region, where the condition $M \ll r-r_{+} \ll 1 / \omega$ is satisfied. The equation for the characteristic value $\omega$ is obtained by imposing suitable boundary conditions at the boundaries. In the following, $r_{+}$denotes the location of the "would-be" horizon. The location of the mirror or ultra-stiff wall is $r_{0}=r_{+}(1+\epsilon), \epsilon \ll 1$.

In the far region, where the effects induced by the BH can be neglected, one can approximate $a \sim 0, M \sim 0$ and $\Delta \sim r^{2}$. The radial wave equation reduces to the wave equation for a massless scalar field of frequency $\omega$ and angular momentum $l$ in flat background

$$
\partial_{r}^{2}(r R)+\left[\omega^{2}-l(l+1) / r^{2}\right](r R)=0 .
$$

The most general solution of this equation is the linear combination of Bessel functions [38]

$$
R=r^{-1 / 2}\left[\alpha J_{l+1 / 2}(\omega r)+\beta J_{-l-1 / 2}(\omega r)\right] .
$$

The behavior of Eq. (A2) for large $r$ and small $r$ are

$$
R \sim \sqrt{\frac{2}{\pi \omega}} \frac{1}{r}[\alpha \sin (\omega r-l \pi / 2)+\beta \cos (\omega r+l \pi / 2)], \quad R \sim \alpha \frac{(\omega / 2)^{l+1 / 2}}{\Gamma(l+3 / 2)} r^{l}+\beta \frac{(\omega / 2)^{-l-1 / 2}}{\Gamma(-l+1 / 2)} r^{-l-1},
$$


respectively. Absence of ingoing waves at infinity implies $\beta=-i \alpha e^{i \pi l}$. The radial wave equation in the near region is

$$
\Delta \partial_{r}\left(\Delta \partial_{r} R\right)+r_{+}^{4}(\omega-m \Omega)^{2} R-l(l+1) \Delta R=0 .
$$

Introducing the radial coordinate

$$
z=\frac{r-r_{+}}{r-r_{-}}, \quad 0 \leq z \leq 1
$$

Eq. (A4) can be rewritten as

$$
z(1-z) \partial_{z}^{2} F+\{(1+i 2 \varpi)-[1+2(l+1)+i 2 \varpi] z\} \partial_{z} F-\left[(l+1)^{2}+i 2 \varpi(l+1)\right] F=0,
$$

where $R=z^{i \varpi}(1-z)^{l+1} F$ and

$$
\varpi \equiv(\omega-m \Omega) \frac{r_{+}^{2}}{r_{+}-r_{-}} .
$$

Equation (A6) is a standard hypergeometric equation. Its most general solution is

$$
R=A z^{-i \varpi}(1-z)^{l+1} F(a-c+1, b-c+1,2-c, z)+B z^{i \varpi}(1-z)^{l+1} F(a, b, c, z),
$$

where $a=1+l+i 2 \varpi, b=l+1$ and $c=1+i 2 \varpi$. Near the wall, where $r \sim r_{+}$, Eq. (A8) reads

$$
R \approx A z^{-i \varpi}+B z^{i \varpi}=A e^{-i(\omega-m \Omega) \frac{r_{+}}{2 M} r_{*}}+B e^{i(\omega-m \Omega) \frac{r_{+}}{2 M} r_{*}},
$$

where $r_{*}$ is the tortoise coordinate

$$
r_{*}=\int \frac{r^{2}+a^{2}}{\left(r-r_{+}\right)\left(r-r_{-}\right)} d r .
$$

The large- $r$ behavior of the solution in the near region is obtained with the change of variable $z \rightarrow 1-z$ in the hypergeometric function [38]. The result is:

$$
\begin{aligned}
R \sim & \left(\frac{r}{r_{+}-r_{-}}\right)^{l} \frac{\Gamma(2 l+1)}{\Gamma(l+1)}\left[A \frac{\Gamma(1-2 i \varpi)}{\Gamma(1+l-2 i \varpi)}+B \frac{\Gamma(1+2 i \varpi)}{\Gamma(l+1+2 i \varpi)}\right]+ \\
& +\left(\frac{r}{r_{+}-r_{-}}\right)^{-l-1} \frac{\Gamma(-1-2 l)}{\Gamma(-l)}\left[A \frac{\Gamma(1-2 i \varpi)}{\Gamma(-l-2 i \varpi)}+B \frac{\Gamma(1+2 i \varpi)}{\Gamma(-l+2 i \varpi)}\right] .
\end{aligned}
$$

The matching of the near- and far-region solutions in the region $M \ll r-r_{+} \ll 1 / \omega$ yields

$$
\frac{B}{A}=-\frac{R_{1}+i(-1)^{l}\left(\omega\left(r_{+}-r_{-}\right)\right)^{2 l+1} L R_{3}}{R_{2}+i(-1)^{l}\left(\omega\left(r_{+}-r_{-}\right)\right)^{2 l+1} L R_{4}}
$$

where

$$
L=\frac{\pi}{2^{2 l+2}} \frac{(\Gamma(l+1))^{2}}{\Gamma(l+3 / 2) \Gamma(2 l+2) \Gamma(2 l+1) \Gamma(l+1 / 2)}
$$

and

$$
R_{1}=\frac{\Gamma(1-2 i \varpi)}{\Gamma(1+l-2 i \varpi)}, \quad R_{2}=\frac{\Gamma(1+2 i \varpi)}{\Gamma(l+1+2 i \varpi)}, \quad R_{3}=\frac{\Gamma(1-2 i \varpi)}{\Gamma(-l-2 i \varpi)}, \quad R_{4}=\frac{\Gamma(1+2 i \varpi)}{\Gamma(-l+2 i \varpi)} .
$$

Equation (A12) can be rewritten as

$$
\frac{B}{A}=-\prod_{k=1}^{l}\left(\frac{k+2 i \varpi}{k-2 i \varpi}\right)\left[\frac{1+2 L\left(r_{+}-r_{-}\right)^{2 l+1} \varpi \omega^{2 l+1} \prod_{k=1}^{l}\left(k^{2}+4 \varpi^{2}\right)}{1-2 L\left(r_{+}-r_{-}\right)^{2 l+1} \varpi \omega^{2 l+1} \prod_{k=1}^{l}\left(k^{2}+4 \varpi^{2}\right)}\right],
$$


where

$$
\begin{gathered}
\frac{R_{1}}{R_{2}}=\prod_{k=1}^{l}\left(\frac{k+2 i \varpi}{k-2 i \varpi}\right), \\
R_{3}=i R_{1}(-1)^{l+1} 2 \varpi \prod_{k=1}^{l}\left(k^{2}+4 \varpi^{2}\right),
\end{gathered}
$$

and similar relations for $R_{4}$ and $R_{2}$ with $\varpi \rightarrow-\varpi$ have been used. If the mirror is located near the outer horizon at a radius $r=r_{0}$, the scalar field must vanish at the mirror surface. This condition implies

$$
\frac{B}{A} z_{0}^{2 i \varpi}=-\frac{F\left(l+1, l+1-2 i \varpi, 1-2 i \varpi, z_{0}\right)}{F\left(l+1, l+1+2 i \varpi, 1+2 i \varpi, z_{0}\right)},
$$

where $z_{0}=z\left(r_{0}\right)$. The relation between the position of the mirror and the frequency of the scalar wave is obtained from Eq. (A15):

$$
\frac{F\left(l+1, l+1-2 i \varpi, 1-2 i \varpi, z_{0}\right)}{F\left(l+1, l+1+2 i \varpi, 1+2 i \varpi, z_{0}\right)}=z_{0}^{2 i \varpi} \prod_{k=1}^{l}\left(\frac{k+2 i \varpi}{k-2 i \varpi}\right)\left[\frac{1+2 L\left(r_{+}-r_{-}\right)^{2 l+1} \varpi \omega^{2 l+1} \prod_{k=1}^{l}\left(k^{2}+4 \varpi^{2}\right)}{1-2 L\left(r_{+}-r_{-}\right)^{2 l+1} \varpi \omega^{2 l+1} \prod_{k=1}^{l}\left(k^{2}+4 \varpi^{2}\right)}\right] .
$$

In general, Eq. A17) must be solved numerically. However, an approximate solution can be obtained by assuming $\operatorname{Re}(\omega) \gg \operatorname{Im}(\omega)$ and $\varpi \ll 1$, i.e. a frequency near the superradiant limit $\omega \approx m \Omega$. This solution gives a good approximation for $M \omega \ll 1$, small $m$ and slowly rotating objects. Since $\operatorname{Re}(\omega) / \operatorname{Im}(\omega) \ll 1$, Eq. (A17) can be first solved for real $\omega$, then a small imaginary part is added and the equation is solved again for $\operatorname{Im}(\omega)$. The l.h.s. and the last two terms of the r.h.s. of Eq. (A17) are $\sim 1$ for frequencies near the superradiant limit. This yields $z_{0}^{2 i \varpi}=1$. Using the tortoise coordinate, it follows

$$
e^{2 i \varpi r_{*}^{0}\left(r_{+}-r_{-}\right) /\left(2 M r_{+}\right)}=e^{2 i \varpi x}=1,
$$

where $r_{*}^{0}=r_{*}\left(z_{0}\right)$ and $x=r_{*}^{0}\left(r_{+}-r_{-}\right) /\left(2 M r_{+}\right)=\log \left(z_{0}\right)$. The solution of Eq. (A18) is

$$
\omega_{n, m}=\frac{\pi\left(r_{+}-r_{-}\right)}{r_{+}^{2} x} n+m \Omega .
$$

Positive frequencies can be obtained by imposing $x<-n \pi\left(r_{+}-r_{-}\right) /\left(m \Omega r_{+}^{2}\right)$. The superradiant limit requires $\varpi=n \pi\left(r_{+}-r_{-}\right) /\left(r_{+}^{2} x\right) \ll 1$. This condition is satisfied by considering only the fundamental tone and the first overtones or placing the mirror very close to the horizon, $|x| \gg 1$. By adding a small imaginary part to the resonant frequency, $\omega=\omega_{n, m}+i \delta$, where $\delta<<\omega_{n, m}$, Eq. (A17) becomes

$$
\begin{aligned}
& \frac{F\left(l+1, l+1-2 i\left(\varpi_{0}+i \rho \delta\right), 1-2 i\left(\varpi_{0}+i \rho \delta\right), z_{0}\right)}{F\left(l+1, l+1+2 i\left(\varpi_{0}+i \rho \delta\right), 1+2 i\left(\varpi_{0}+i \rho \delta\right), z_{0}\right)} z_{0}{ }^{-2 i \varpi_{0}} z_{0}^{2 \rho \delta}= \\
& =\prod_{k=1}^{l}\left(\frac{k+2 i \varpi_{0}}{k-2 i \varpi_{0}}\right)\left[\frac{1+2 L\left(r_{+}-r_{-}\right)^{2 l+1} \varpi_{0} \omega_{n, m}^{2 l+1} \prod_{k=1}^{l}\left(k^{2}+4 \varpi_{0}^{2}\right)}{1-2 L\left(r_{+}-r_{-}\right)^{2 l+1} \varpi_{0} \omega_{n, m}^{2 l+1} \prod_{k=1}^{l}\left(k^{2}+4 \varpi_{0}^{2}\right)}\right],
\end{aligned}
$$

where $\varpi=\varpi_{0}+i \rho \delta$ and $\rho=r_{+}^{2} /\left(r_{+}-r_{-}\right)$. The ratio of the hypergeometric functions in the l.h.s. of Eq. (A20) is $\sim 1$ for the approximations used in the derivation. In terms of the tortoise coordinate, Eq. (A20) reads

$$
e^{2 \rho x \delta}=\frac{1+2 L\left(r_{+}-r_{-}\right)^{2 l+1} \varpi_{0} \omega_{n, m}^{2 l+1} \prod_{k=1}^{l}\left(k^{2}+4 \varpi_{0}^{2}\right)}{1-2 L\left(r_{+}-r_{-}\right)^{2 l+1} \varpi_{0} \omega_{n, m}^{2 l+1} \prod_{k=1}^{l}\left(k^{2}+4 \varpi_{0}^{2}\right)} .
$$

The solution of Eq. A21) is

$$
\delta=\operatorname{Im}(\omega)=\frac{r_{+}-r_{-}}{2 r_{+}^{2} x} \log \left[\frac{1+\gamma\left(\omega_{n, m}-m \Omega\right)}{1-\gamma\left(\omega_{n, m}-m \Omega\right)}\right]
$$


where

$$
\gamma \equiv \frac{\pi}{2} r_{+}^{2}\left(\frac{r_{+}-r_{-}}{2}\right)^{2 l} \prod_{k=1}^{l}\left(k^{2}+4 \varpi_{0}^{2}\right) \frac{[\Gamma(l+1)]^{2}}{\Gamma(l+3 / 2) \Gamma(2 l+2) \Gamma(2 l+1) \Gamma(l+1 / 2)} \omega_{n, m}^{2 l+1} \geq 0 .
$$

Both $\delta$ and $\omega_{n, m}$ are very small for $r_{0} \sim r_{+}$. However, the argument of the logarithm in Eq. (A22) is $\sim 1$ and the assumption $\operatorname{Re}(\omega) \gg \operatorname{Im}(\omega)$ is satisfied.

The above results display two important features. First, Eq. (A22) and Eq. (A23) imply $\delta \lessgtr 0$ for $\operatorname{Re}(\omega) \gtrless m \Omega$. The time dependence of the scalar field $\Phi$ is $\exp (-i \omega t)=\exp (-i \operatorname{Re}(\omega) t) \exp (\delta t)$. Thus the amplitude of the field grows exponentially and the resonant mode becomes instable for $\operatorname{Re}(\omega)<m \Omega$. Second, $x<x_{\text {crit }}=-n \pi\left(r_{+}-r_{-}\right) /\left(m \Omega r_{+}^{2}\right)$ and $\operatorname{Re}\left(\varpi_{0}\right) \propto n x^{-1}$ imply $\delta \lessgtr 0$ for $n \lessgtr 0$. There is always a superradiant amplification for $n>0$ provided that the mirror position is closer than $x_{c r i t}$ to the horizon and the approximations used in the above derivation are satisfied. The critical value $x_{\text {crit }}$ is positive and outside the domain of $x$ for $n<0$. In this case the mirror can be located everywhere in the near region, but there is no superradiant amplification. The growth timescale for $n>0$ is given by

$$
\tau \equiv \delta^{-1}=2 \rho x \log ^{-1}\left[\frac{1+\gamma\left(\omega_{n, m}-m \Omega\right)}{1-\gamma\left(\omega_{n, m}-m \Omega\right)}\right]
$$

or, in terms of the physical variables, by Eq. (3.6).

\section{Massive scalar field}

If the scalar field is massive, the wave equation is

$$
\Delta_{\mu} \Delta^{\mu} \Psi=\mu^{2} \Psi
$$

where $\Delta_{\mu}$ the covariant derivative and $\mu$ is the field mass. The above equation is separable. The radial equation is

$$
\Delta \frac{d}{d r}\left(\Delta \frac{d R}{d r}\right)+\left[\omega^{2}\left(r^{2}+a^{2}\right)^{2}-4 a M \omega r++a^{2} m^{2}-\Delta\left(\mu 2 r^{2}+a^{2} \omega^{2}+\lambda\right)\right] R=0 .
$$

Assuming $a \omega \ll 1, \lambda \approx l(l+1)$ and $\mu r \ll l$, the solution in the near region is identical to the solution for the massless case. The equation in the far region is

$$
\partial_{r}^{2}(r R)+\left[\omega^{2}-\mu^{2}-l(l+1) / r^{2}\right](r R)=0 .
$$

The results of the massless case apply with the substitution $\omega \rightarrow k$, where $k^{2}=\omega^{2}-\mu^{2}>0$. The matching conditions are

$$
\frac{F\left(l+1, l+1-2 i \varpi, 1-2 i \varpi, z_{0}\right)}{F\left(l+1, l+1+2 i \varpi, 1+2 i \varpi, z_{0}\right)}=z_{0}^{2 i \varpi} \prod_{k=1}^{l}\left(\frac{k+2 i \varpi}{k-2 i \varpi}\right)\left[\frac{1+2 L\left(r_{+}-r_{-}\right)^{2 l+1} \varpi\left(\omega^{2}-\mu^{2}\right)^{l+1 / 2} \prod_{k=1}^{l}\left(k^{2}+4 \varpi^{2}\right)}{1-2 L\left(r_{+}-r_{-}\right)^{2 l+1} \varpi\left(\omega^{2}-\mu^{2}\right)^{l+1 / 2} \prod_{k=1}^{l}\left(k^{2}+4 \varpi^{2}\right)}\right] .
$$

The imaginary part of the frequency is identical to Eq. (A22) with

$$
\gamma \equiv \pi r_{+}^{2}\left(\frac{r_{+}-r_{-}}{2}\right)^{2 l} \prod_{k=1}^{l}\left(k^{2}+4 \varpi_{0}^{2}\right)\left[\frac{[\Gamma(l+1)]^{2}}{\Gamma(l+3 / 2) \Gamma(2 l+2) \Gamma(2 l+1) \Gamma(l+1 / 2)}\right]\left(\omega_{n, m}^{2}-\mu^{2}\right)^{l+1 / 2} \geq 0 .
$$

The condition for superradiant amplification, $\omega<m \Omega$, does not depend on the field mass.

\section{General spin-s fields}

Previous analytical and numerical calculations [25, 39] have shown that superradiant effects for gravitational fields are stronger than for scalar fields. For instance, superradiant amplification factors are about $0.1 \%, 4.5 \%$ and $138 \%$ for scalar, electromagnetic and gravitational field, respectively. Since the effects induced by the BH in the far region can be neglected, the radial wave equation reduces to the wave equation of a massless field with spin-weight $s$, frequency $\omega$ and angular momentum $l$ in flat background:

$$
r \partial_{r}^{2} f+2(1+l-i \omega r) \partial_{r} f-2 i(1+l-s) \omega f=0,
$$


where $R=e^{-i \omega r} r^{l-s} f(r)$. Introducing the radial coordinate $x=2 i \omega r$, the wave equation becomes a standard Kummer equation [38]. Its most general solution is a linear combination of confluent hypergeometric functions:

$$
R=e^{-i \omega r} r^{l-s}(\alpha M(1+l-s, 2 l+2,2 i \omega r)+\beta U(1+l-s, 2 l+2,2 i \omega r)) .
$$

The large- $r$ behavior of Eq. (A30) is

$$
R \sim\left[\alpha \frac{\Gamma(2 l+2)}{\Gamma(1+l-s)}(2 i \omega)^{-1-l-s}\right] \frac{e^{i \omega r}}{r^{1+2 s}}+\left[\alpha(-1)^{1+l-s} \frac{\Gamma(2 l+2)}{\Gamma(1+l+s)}+\beta\right] \frac{e^{-i \omega r}}{r} .
$$

The first two terms in Eq. (A31) represent an outgoing wave at infinity and an incoming wave from infinity, respectively. The behavior for small $r$ is

$$
R \sim\left[\alpha+\beta \frac{\Gamma(-1-2 l)}{\Gamma(-l-s)}\right] r^{l-s}+\beta(2 i \omega)^{-1-2 l} \frac{\Gamma(2 l+1)}{\Gamma(1+l-s)} r^{-1-l-s} .
$$

Absence of ingoing waves at infinity implies

$$
\beta=-\alpha(-1)^{1+l-s} \frac{\Gamma(2 l+2)}{\Gamma(1+l+s)} .
$$

The near-region behavior of the solution in the far region is

$$
R \sim \alpha\left[\frac{1}{2} r^{l-s}-(2 i \omega)^{-1-2 l}(-1)^{1+l-s} \frac{\Gamma(2 l+2) \Gamma(2 l+1)}{\Gamma(1+l-s) \Gamma(1+l+s)} r^{-l-1-s}\right] .
$$

The radial wave equation in the near region is

$$
\Delta^{-s} \frac{d}{d r}\left(\Delta^{s+1} \frac{d R}{d r}\right)+\left[\frac{K^{2}-2 i s(r-M) K}{\Delta}-\lambda\right] R=0,
$$

where $\lambda=(l-s)(l+s+1)+O(\omega a)$. Using the approximate relations

$$
\Delta^{\prime} \approx r_{+}-r_{-}, \quad K=\omega\left(r^{2}+a^{2}\right)-a m \approx r_{+}^{2}(\omega-m \Omega), \quad K^{2}-2 i s(r-M) K \approx\left(r_{+}-r_{-}\right)^{2}\left[\frac{s^{2}}{4}+\left(\varpi-i \frac{s}{2}\right)^{2}\right],
$$

and introducing the radial coordinate $z$, Eq. (A35) can be written as

$$
z(1-z) \partial_{z}^{2} R+((s+1)+(s-1) z) \partial_{z} R+\left\{\frac{1-z}{z}\left[\frac{s^{2}}{4}+(\varpi-i s / 2)^{2}\right]-\frac{(l-s)(l+s+1)}{1-z}\right\} R=0 .
$$

Setting $R=z^{i \varpi}(1-z)^{l+s+1} F$, the previous equation becomes a standard hypergeometric equation [38]. Its most general solution is

$$
R=A z^{-s-i \varpi}(1-z)^{l+1+s} F(a-c+1, b-c+1,2-c, z)+B z^{i \varpi}(1-z)^{l+1+s} F(a, b, c, z),
$$

where $a=1+l+s+i 2 \varpi, b=l+1$ and $c=1+s+i 2 \varpi$. The behavior of Eq. (A36) near $r_{+}$is

$$
R \sim A e^{-s-i(\omega-m \Omega) \frac{r_{+}}{2 M} r_{*}}+B e^{i(\omega-m \Omega) \frac{r_{+}}{2 M} r_{*}} .
$$

The large- $r$ behavior is

$$
R \sim\left(\frac{r}{r_{+}-r_{-}}\right)^{l-s} \frac{\Gamma(2 l+1)}{\Gamma(l+1)}\left(A R_{1}+B R_{2}\right)+\left(\frac{r}{r_{+}-r_{-}}\right)^{-l-1-s} \frac{\Gamma(-1-2 l)}{\Gamma(-l)}\left(A R_{3}+B R_{4}\right),
$$

where

$$
\begin{aligned}
& R_{1}=\frac{\Gamma(1-s-2 i \varpi)}{\Gamma(l-s+1-2 i \varpi)}, \quad R_{2}=\frac{\Gamma(1+s+2 i \varpi)}{\Gamma(l+s+1+2 i \varpi)}, \\
& R_{3}=\frac{\Gamma(1-s-2 i \varpi)}{\Gamma(-l-s-2 i \varpi)}=R_{1}(-1)^{l+1}(s+2 i \varpi) \prod_{k=1}^{l}\left(k^{2}+4 \varpi^{2}-s^{2}-4 i s \varpi\right), \\
& R_{4}=\frac{\Gamma(1+s+2 i \varpi)}{\Gamma(-l+s+2 i \varpi)}=-R_{2}(-1)^{l+1}(s+2 i \varpi) \prod_{k=1}^{l}\left(k^{2}+4 \varpi^{2}-s^{2}-4 i s \varpi\right) .
\end{aligned}
$$


The matching of Eq. (A38) and Eq. (A34) yields

$$
\frac{B}{A}=-\prod_{k=1}^{l}\left(\frac{k+s+2 i \varpi}{k-s-2 i \varpi}\right) \frac{1-i \gamma L_{s}(s+2 i \varpi) g_{s}}{1+i \gamma L_{s}(s+2 i \varpi) g_{s}}
$$

where $\gamma=\left(w\left(r_{+}-r_{-}\right)\right)^{2 l+1}, g_{s}=\prod_{k=1}^{l}\left(k^{2}+4 \varpi^{2}-s^{2}-4 i s \varpi\right)$ and $L_{s}$ is defined in Eq. (3.5) for $l \neq \pm s-1$. Equation (A40) reduces to Eq. (A15) for $s=0$.

If the mirror is close to the outer horizon, the field must vanish at the mirror surface. Using the small- $r$ behavior of the solution in the near region and setting the radial field to zero at $z=z_{0}$, it follows $A=-B z_{0}^{s+2 i \varpi}$. The relation between the position of the mirror and the frequency of the wave is given by Eq. (3.4).

\section{APPENDIX B: ANALYTIC SOLUTION IN THE NEAR-EXTREMAL REGIME}

This appendix describes near-extremal horizonless objects by employing the analytic approximations of Refs. 255, 39]. The resonant frequencies for rapidly spinning BHs can be found by rewriting the Teukolsky equation in the Kerr incoming coordinate system $\left(v, r, \theta, \phi^{\prime}\right)$. The latter is obtained from the Boyer-Lindquist coordinates with the coordinate transformation $d v=d t+\left(r^{2}+a^{2}\right) d r / \Delta, d \phi^{\prime}=d \phi+a d r / \Delta$. The radial equation for a field of spin $s$ is [39]

$$
\Delta R^{\prime \prime}(r)+2[(s+1)(r-M)-i K] R^{\prime}(r)-[2(2 s+1) i \omega r-\lambda] R(r)=0 .
$$

Defining $x=\left(r-r_{+}\right) / r_{+}, \sigma=\left(r_{+}-r_{-}\right) / r_{+}, \tau=M(\omega-m \Omega)$ and $\omega^{\prime} \rightarrow r_{+} \omega$, Eq. (B1) reads

$$
x(x+\sigma) R^{\prime \prime}(x)-\left[2(2 s+1) i \omega^{\prime}(x+1)+\lambda\right] R(x)-\left[2 i \omega^{\prime} x^{2}+x\left(4 i \omega^{\prime}-2(s+1)\right)-(s+1) \sigma++4 i \tau\right] R^{\prime}(x)=0 .
$$

In the far region, $x \gg \max (\sigma, \tau)$, Eq. (B2) reduces to

$$
x^{2} R^{\prime \prime}(x)-\left[2 i \omega^{\prime} x^{2}+x\left(4 i \omega^{\prime}-2(s+1)\right)\right] R^{\prime}(x)-\left[2(2 s+1) i \omega^{\prime}(x+1)+\lambda\right] R(x)=0 .
$$

Its most general solution is written in terms of confluent hypergeometric functions

$$
R(x)=\alpha x^{-s-\frac{1}{2}+2 i \omega^{\prime}+i \delta} M\left(\frac{1}{2}+s+2 i \omega^{\prime}+i \delta, 1+2 i \delta, 2 i \omega x\right)+\beta(\delta \rightarrow-\delta),
$$

where $\delta^{2}=4 \omega^{\prime 2}-(s+1 / 2)^{2}-\lambda$. The large- $x$ behavior of Eq. (B4) is

$$
R \sim x^{-1-2 s}\left[\frac{\alpha \Gamma(1+2 i \delta)\left(e^{i \pi} / 2 i \omega^{\prime}\right)^{1 / 2+s+2 i \omega^{\prime}+i \delta}}{\Gamma\left(1 / 2-s-2 i \omega^{\prime}+i \delta\right)}\right]+\frac{e^{2 i \omega^{\prime} x}}{x^{1-4 i \omega^{\prime}}}\left[\frac{\alpha \Gamma(1+2 i \delta)\left(2 i \omega^{\prime}\right)^{-1 / 2+s+2 i \omega^{\prime}-i \delta}}{\Gamma\left(1 / 2+s+2 i \omega^{\prime}+i \delta\right)}\right]+\beta(\delta \rightarrow-\delta) .
$$

The term representing an incoming (outgoing) wave at infinity behaves as $r^{-1-2 s}=e^{2 i \omega r_{*}} / r$ for near-extremal BHs. (See Table 1 of Ref. [39]). Thus the first and second terms in Eq. (B5) describe an incoming wave and an outcoming wave, respectively. Absence of incoming waves at infinity implies

$$
\beta=-\alpha \frac{\Gamma(1+2 i \delta)}{\Gamma(1-2 i \delta)} \frac{\Gamma\left(1 / 2-s-2 i \omega^{\prime}-i \delta\right)}{\Gamma\left(1 / 2-s-2 i \omega^{\prime}+i \delta\right)}\left(-2 i \omega^{\prime}\right)^{-2 i \delta} .
$$

Equation (B4) reduces to

$$
R \sim \alpha x^{-s-1 / 2+2 i \omega^{\prime}+i \delta}+\beta x^{-s-1 / 2+2 i \omega^{\prime}-i \delta}
$$

for small $r$. In the near region, Eq. (B2) reads

$$
x(x+\sigma) R^{\prime \prime}(x)-\left[x\left(4 i \omega^{\prime}-2(s+1)\right)-(s+1) \sigma+4 i \tau\right] R^{\prime}(x)+\left[2(2 s+1) i \omega^{\prime}+\lambda\right] R(x)=0 .
$$

The radial wave equation in the near region becomes a standard hypergeometric equation with the substitution $z=-x / \sigma$. Its most general solution is

$$
R=A z^{1-c} F(a-c+1, b-c+1,2-c, z)+B F(a, b, c, z),
$$


where $a=1 / 2+s-2 i \omega^{\prime}+i \delta, b=1 / 2+s-2 i \omega^{\prime}-i \delta, c=1+s+i \kappa$ and $\kappa=-4 \tau / \sigma$. The mirror is located at $r \sim r_{+}$. In this limit the solution behaves as $R \sim A z^{1-c}+B$. The large- $r$ behavior of the solution in the near region is

$$
\begin{aligned}
R \sim\left(\frac{x}{\sigma}\right)^{-a}\left[A(-1)^{1-c} \frac{\Gamma(2-c) \Gamma(b-a)}{\Gamma(b-c+1) \Gamma(1-a)}+B \frac{\Gamma(c) \Gamma(b-a)}{\Gamma(b) \Gamma(c-a)}\right]+ \\
+\left(\frac{x}{\sigma}\right)^{-b}\left[A(-1)^{1-c} \frac{\Gamma(2-c) \Gamma(a-b)}{\Gamma(a-c+1) \Gamma(1-b)}+B \frac{\Gamma(c) \Gamma(a-b)}{\Gamma(a) \Gamma(c-b)}\right],
\end{aligned}
$$

or, in terms of the physical variables,

$$
R \sim\left(\frac{x}{\sigma}\right)^{-1 / 2-s+2 i \omega^{\prime}+i \delta} \Gamma(2 i \delta)\left[A(-1)^{s+i \kappa} R_{1}+B R_{2}\right]+(\delta \rightarrow-\delta),
$$

where

$$
\begin{aligned}
& R_{1}(\delta)=\frac{\Gamma(1-s-i \kappa)}{\Gamma\left(1 / 2-s+2 i \omega^{\prime}+i \delta\right) \Gamma\left(1 / 2-2 i \omega^{\prime}+i \delta-i \kappa\right)}, \\
& R_{2}(\delta)=\frac{\Gamma(1+s+i \kappa)}{\Gamma\left(1 / 2+s-2 i \omega^{\prime}+i \delta\right) \Gamma\left(1 / 2+2 i \omega^{\prime}+i \delta+i \kappa\right)}, \\
& R_{3}=R_{1}(-\delta), \quad R_{4}=R_{2}(-\delta) .
\end{aligned}
$$

The matching in the overlapping region yields

$$
\alpha=\sigma^{b} \Gamma(a-b)\left[A(-1)^{1-c} R_{1}+B R_{2}\right], \quad \beta=\sigma^{a} \Gamma(b-a)\left[A(-1)^{1-c} R_{3}+B R_{4}\right] .
$$

Using Eq. (B6), it follows

$$
-\rho\left(-2 i \omega^{\prime} \sigma\right)^{2 i \delta}=\frac{A(-1)^{s+i \kappa} R_{1}+B R_{2}}{A(-1)^{s+i \kappa} R_{3}+B R_{4}}
$$

where

$$
\rho=\frac{\Gamma(-2 i \delta) \Gamma(1-2 i \delta) \Gamma\left(1 / 2-s-2 i \omega^{\prime}+i \delta\right)}{\Gamma(2 i \delta) \Gamma(1+2 i \delta) \Gamma\left(1 / 2-s-2 i \omega^{\prime}-i \delta\right)} .
$$

Equation (B13) reduces to Eq. (9) of Ref. [40] for suitable boundary condition in presence of a horizon, i.e. $A=0$. In the case under study, $A$ is nonvanishing because the region containing the horizon is forbidden by the presence of the mirror. Thus Eq. (B12) yields

$$
\frac{B}{A}=-(-1)^{s+i \kappa} \frac{R_{1}+\rho R_{3}\left(-2 i \sigma \omega^{\prime}\right)^{2 i \delta}}{R_{2}+\rho R_{4}\left(-2 i \sigma \omega^{\prime}\right)^{2 i \delta}}
$$

Dirichlet boundary conditions at the wall require $z_{0}^{-s-i \kappa}=-B / A$. The relation between the position of the mirror and the frequency of the wave, Eq. (3.8), is obtained from Eq. (B14).

[1] C. F. Gammie, S. L. Shapiro and J. C. McKinney, Astrophys. J. 602, 312 (2004).

[2] D. Merritt and M. Milosavljevic, "Massive black hole binary evolution," arXiv:astro-ph/0410364.

[3] J. M. Wang, Y. M. Chen, L. C. Ho and R. J. McLure, Astrophys. J. 642, L111 (2006).

[4] R. Narayan, New J. Phys. 7, 199 (2005).

[5] M. A. Abramowicz, W. Kluzniak and J. P. Lasota, Astron. Astrophys. 396, L31 (2002).

[6] J. P. Lasota, Comptes Rendus Physique 8, 45 (2007).

[7] G. Chapline, E. Hohlfeld, R. B. Laughlin and D. I. Santiago, Int. J. Mod. Phys. A 18, 3587 (2003).

[8] P. O. Mazur and E. Mottola, "Gravitational condensate stars," arXiv:gr-qc/0109035

[9] D. J. Kaup, Phys. Rev. 172, 1331 (1968); R. Ruffini and S. Bonazzola, Phys. Rev. 187, 1767 (1969).

[10] E. Berti and V. Cardoso, Int. J. Mod. Phys. D 15, 2209 (2006).

[11] V. Cardoso, P. Pani, M. Cadoni and M. Cavaglià, Phys. Rev. D in press, arXiv:0709.0532 [gr-qc].

[12] M. S. Morris and K. S. Thorne, Am. J. Phys. 56, 395 (1988).

[13] M. Visser, Lorentzian wormholes: From Einstein to Hawking, (American Institute of Physics, NY, 1995). 
[14] J. P. S. Lemos, F. S. N. Lobo and S. Quinet de Oliveira, Phys. Rev. D 68, 064004 (2003).

[15] T. Damour and S. N. Solodukhin, Phys. Rev. D 76, 024016 (2007).

[16] S. D. Mathur, Fortsch. Phys. 53, 793 (2005).

[17] E. G. Gimon and P. Horava, "Astrophysical violations of the Kerr bound as a possible signature of string theory," arXiv:0706.2873 [hep-th].

[18] G. E. A. Matsas and A. R. R. da Silva, Phys. Rev. Lett. 99, 181301 (2007)-

[19] W. H. Press and S. A. Teukolsky, Nature 238, 211 (1972).

[20] V. Cardoso, O. J. C. Dias, J. P. S. Lemos and S. Yoshida, Phys. Rev. D 70, 044039 (2004) [Erratum-ibid. D 70, 049903 (2004)]; V. Cardoso and O. J. C. Dias, Rev. D 70, 084011 (2004);

[21] N. Drukker, Phys. Rev. D 70, 084031 (2004).

[22] E. Teo, Phys. Rev. D 58, 024014 (1998).

[23] S. A. Teukolsky, Phys. Rev. Lett 29, 1114 (1972); S. A. Teukolsky, Astrophys. J. 185, 635 (1973).

[24] W. G. Unruh, Phys. Rev. D 14, 3251 (1976).

[25] A. A. Starobinsky, Zh. Eksp. Teor. Fiz 64, 48 (1973) [Sov. Phys. JETP 37, 28 (1973)]; A. A. Starobinsky and S. M. Churilov, Zh. Eksp. Teor. Fiz 65, 3 (1973) [Sov. Phys. JETP 38, 1 (1973)].

[26] S. W. Hawking, Commun. Math. Phys. 43, 199 (1975) [Erratum-ibid. 46, 206 (1976)].

[27] E. Berti, V. Cardoso and M. Casals, Phys. Rev. D 73, 024013 (2006) [Erratum-ibid. D 73, 109902 (2006).

[28] R. Emparan and R. C. Myers, JHEP 0309, 025 (2003).

[29] R. Gregory and R. Laflamme, Phys. Rev. Lett. 70, 2837 (1993).

[30] V. Cardoso and O. J. C. Dias, Phys. Rev. Lett. 96, 181601 (2006); V. Cardoso and L. Gualtieri, Class. Quant. Grav. 23, 7151 (2006); V. Cardoso, O. J. C. Dias and L. Gualtieri, "The return of the membrane paradigm? Black holes and strings in the water tap," arXiv:0705.2777 [hep-th].

[31] R. J. Gleiser and G. Dotti, Class. Quant. Grav. 23, 5063 (2006).

[32] V. Cardoso and M. Cavaglià, Phys. Rev. D 74, 024027 (2006).

[33] G. Dotti, R. Gleiser and J. Pullin, Phys. Lett. B 644, 289 (2007).

[34] S. Chandrasekhar, Proc. R. Soc. London, Ser. A 392, 1 (1984); The Mathematical Theory of Black Holes, (Oxford University Press, New York, 1983).

[35] G. Dotti, R. J. Gleiser, I. F. Ranea-Sandoval and H. Vucetich, arXiv:0805.4306] [gr-qc].

[36] E. W. Leaver, Proc. Roy. Soc. Lond. A 402, 285 (1985).

[37] H. Onozawa, Phys. Rev. D 55, 3593 (1997).

[38] M. Abramowitz and A. Stegun, Handbook of mathematical functions, (Dover Publications, New York, 1970).

[39] S. A. Teukolsky and W. H. Press, Astrophys. J. 193, 443 (1974).

[40] S. Detweiler, Astrophys. J. 239, 292 (1980). 